\title{
Post-keratoplasty endophthalmitis caused by Proteus mirabilis
}

\section{Abstract}

A diabetic patient who underwent a triple procedure (penetrating keratoplasty, cataract extraction and posterior chamber intraocular lens implantation), developed endophthalmitis caused by Proteus mirabilis. The source of infection was the infected donor cornea, which was imported from Sri Lanka. The organism was resistant to gentamicin, which was the only antibiotic present in the storage medium. On top of an aggressive antibiotic treatment regime (topical, systemic and intravitreal), exchange of the infected with a fresh corneal graft and pars plana vitrectomy were performed. The patient had a speedy recovery and the visual outcome was $20 / 40$ two and a half years after the incident. To our knowledge, post-keratoplasty endophthalmitis caused by $P$. mirabilis has never been reported in the literature. We report herein such a case with good visual outcome. Newer storage medium, such as Optisol GS, might have a role in preventing keratoplasty-related infection.

Key words Penetrating keratoplasty, Endophthalmitis, Proteus mirabilis

The rate of bacterial endophthalmitis complicating penetrating keratoplasty is approximately $0.11-0.8 \% .{ }^{1,2}$ Up to 1993 , fortyfive cases of bacterial endophthalmitis after keratoplasty were reported in the literature. ${ }^{3,4}$ Seventy-eight per cent of these cases had a visual outcome of counting fingers or worse, and only $4 \%$ ( 2 cases) had a visual outcome of $20 / 40$ or better. We report herein a case of Proteus mirabilis endophthalmitis complicating penetrating keratoplasty. The patient has a best corrected visual acuity of 20/40 two and a half years after the incident of endophthalmitis. To our knowledge, this is the first report of post-keratoplasty endophthalmitis caused by P. mirabilis.
DENNIS S.C. LAM, ALVIN K.H. KWOK, SEKJIN CHEW

\section{Case report}

A 74-year-old woman with bilateral interstitial keratitis underwent an uneventful triple procedure (penetrating keratoplasty + extracapsular cataract extraction + posterior chamber intraocular lens implantation) on her right eye in October 1994. At the end of the operation, subconjunctival gentamicin $(20 \mathrm{mg})$ was given. Topical chloramphenicol and hydrocortisone (Pred Forte, Allergan) were used post-operatively. The donor was a 29-year-old man who had died of herbicide poisoning in Sri Lanka. The cornea was harvested within $4 \mathrm{~h}$ of his death. The graft was stored in the MK medium for 3 days before use. On post-operative day 7 , the graft was clear and the unaided visual acuity was $20 / 200$.

On post-operative day 9 , the patient returned with moderate right eye pain and visual acuity that had dropped to hand movement only. The graft was oedematous with diffuse stromal infiltration. There was a $1 \mathrm{~mm}$ hypopyon with $3+$ cells and $3+$ flare in the anterior chamber. Fundal view was blocked by a thick pupillary fibrinous membrane. Ultrasonography (B-scan) showed only a minimal amount of abnormal echo in the anterior one-fifth of the vitreous. The mid- and posterior vitreous and the retina appeared to be normal. Aqueous and vitreous taps were performed immediately. Urgent Gram staining of specimens from both taps revealed only $2+$ white blood cells and no organism. Intravitreal vancomycin $(1 \mathrm{mg})$ and gentamicin $(0.2 \mathrm{mg})$ were given. The patient was further treated with intravenous (vancomycin $1 \mathrm{~g} \mathrm{Q} 12 \mathrm{H}$, gentamicin $60 \mathrm{mg}$ $\mathrm{Q} 8 \mathrm{H}$ ), subconjunctival (vancomycin $25 \mathrm{mg}$, gentamicin $20 \mathrm{mg}$ ) and topical (gutt. vancomycin $50 \mathrm{mg} / \mathrm{ml}$, gentamicin $30 \mathrm{mg} / \mathrm{ml}$ $\mathrm{Q} 1 \mathrm{H})$ antibiotics.

Tracing of the culture report of the donor corneal rim revealed positive growth of Proteus mirabilis, Pseudomonas aeruginosa, Staphylococcus aureus and Citrobacter species. The first two organisms were also grown from the donor

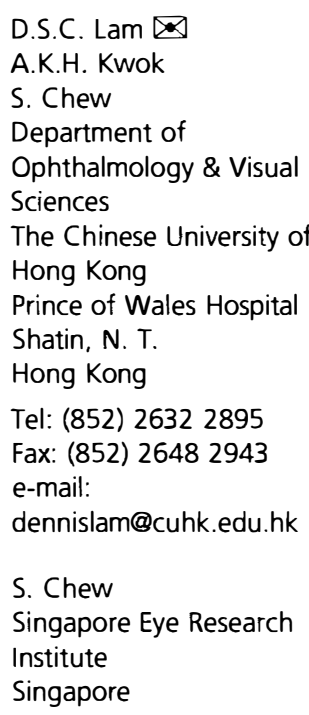

D.S.C. Lam

Department of

Ophthalmology \& Visual

The Chinese University of Hong Kong

Prince of Wales Hospital

Shatin, N. T.

Tel: (852) 26322895

e-mail:

Singapore Eye Research

Singapore 
storage medium. All were gentamicin-resistant but sensitive to amikacin. The antibiotic regime (systemic and topical) was modified accordingly, with substitution of gentamicin by amikacin. The clinical condition deteriorated in the ensuing $36 \mathrm{~h}$ with development of multiple small stromal abscesses in the corneal graft. Repeated B-scan ultrasonography revealed a substantial amount of abnormal echo in the mid-and posterior vitreous.

With the progression and extension of the endophthalmitis, an emergency operation was performed. Intraoperatively, the badly infected donor cornea and the thick inflammatory membrane at the pupillary plane were removed. The intraocular lens and capsules were found to be packed with opaque infected and inflamed material. They were also removed. Open sky anterior vitrectomy as well as limited core vitrectomy were then performed. A new corneal graft was then implanted, followed by a standard three-port pars plana vitrectomy. Intravitreal amikacin $(0.4 \mathrm{mg})$ was given at the end of the operation. Culture of the aqueous fluid, anterior chamber inflammatory membrane, intraocular lens and vitreous fluid all showed positive growth of Proteus mirabilis. The patient then enjoyed an uneventful recovery with best corrected visual acuity of 20/40 at the latest follow-up, which was two and a half years after the operation.

\section{Discussion}

Proteus mirabilis is a Gram-negative facultative anaerobic non-spore-forming rod. ${ }^{5}$ It is present in soil and water as well as human intestinal flora. It usually causes infection only when the host is immunocompromised. Our patient was a diabetic whose immune defence was further compromised by post-operative systemic and topical steroids. $P$. mirabilis endophthalmitis after intraocular surgery is rare. In the nosocomial endophthalmitis survey published by Kattan et al. ${ }^{1}$ only three such cases were reported. One case had a final visual acuity of $20 / 100$ while the other two were not mentioned. It is uncommon for Proteus to be gentamicin-resistant, though multiple reports of resistant strains have been published. ${ }^{6}$

It is strongly advised that routine culture be carried out for the corneal rim and storage medium of donor corneas. Results obtained may help an earlier diagnosis to be made, as well as identifying the source of infection and guiding the antibiotic treatments. The clinical picture and the culture results strongly indicated that the donor cornea was the source of infection. As pointed out by Kaufman et al., ${ }^{7}$ most of the organisms causing postkeratoplasty endophthalmitis are gentamicin resistant. Our patient is a good example. However, until recently gentamicin was the only antibiotic included in commercially available corneal storage medium. The newer corneal storage media such as Optisol GS, with streptomycin added in, may contribute to the prevention of keratoplasty-related infection. ${ }^{8,9}$ In this patient, the donor corneal button was retrieved from Sri Lanka. A significantly higher incidence of endophthalmitis was noted in patients receiving corneas from a Sri Lankan eye bank $(1.23 \%)$ than in those receiving eye bank tissue from the West $(0.14 \%) .{ }^{10}$ It would be ideal to have an international regulatory body advising standard procedures of corneal tissue harvesting and handling prior to dispatch.

In this patient, the endophthalmitis developed 9 days after surgery. The delayed onset suggested the organism might be of a less virulent nature, which contributed to the favourable visual outcome. In the management of pseudophakic bacterial endophthalmitis, intraocular lens and capsules may be left behind. ${ }^{11}$ In our case, we found the intraocular lens and capsules to be very badly infected and we decided to remove them. Whether this contributed to a more favourable outcome is debatable and the option of leaving lens and capsules behind is always open.

\section{References}

1. Kattan HM, Flynn HW Jr, Pfengfelder SC, Robertson C, Forster RK. Nosocomial endophthalmitis survey: current incidence of infection after intraocular surgery. Ophthalmology 1991;98:227-38.

2. Leveille AS, McMullin FD, Cavanagh HD. Endophthalmitis following penetrating keratoplasty. Ophthalmology 1983;90:38-9.

3. Kloess PM, Stulting RD, Waring GO III, Wilson LA. Bacterial and fungal endophthalmitis after penetrating keratoplasty. Am J Ophthalmol 1993;115:309-16.

4. Lopez PF, Beldavs RA, Al-ghamdi Saeed, Wilson LA, Wojno $\mathrm{TH}$, Sternberg P Jr, Aaberg TM, Lambert HM. Pneumococcal endophthalmitis associated with nasolacrimal obstruction. Am J Ophthalmol 1993;116:56-62.

5. Guentzel MN. Escherichia, Klebsiella, Enterobacter, Serratia, Citrobacter, and Proteus. In: Barron S, editor. Medical microbiology. 3rd ed. London: Churchill Livingstone, 1991:377-87.

6. Dance DA, Pearson AD, Seal DV, Lowes JA. A hospital outbreak caused by a chlorhexidine and antibiotic-resistant Proteus mirabilis. J Hosp Infect 1987;10:10-6.

7. Kaufman HE, Barron BA, McDonald MB, Waltman SR. The cornea, 1st ed. London: Churchill Livingstone, 1988.

8. Smith TM, Popplewell J, Nakamura T, Trousdale MD. Efficacy and safety of gentamicin and streptomycin in Optisol GS, a preservation medium for donor corneas. Cornea 1995;14:49-55.

9. Morlet N, Matheson M, Tuft S. Investigation of the antibacterial properties of the Optisol GS. Invest Ophthalmol Vis Sci 1997;38:S861.

10. Cameron JA, Antonios SR, Cotter JB, Habash NR. Endophthalmitis from contaminated donor corneas following penetrating keratoplasty. Arch Ophthalmol 1991;100:54-9.

11. Endophthalmitis Vitrectomy Study Group. Results of the endophthalmitis vitrectomy study, a randomised trial of immediate vitrectomy and of intravenous antibiotics for the treatment of postoperative bacterial endophthalmitis. Arch Ophthalmol 1995;113:1479-96. 